\title{
Design Advances and New Results of a sub-Ångström Dedicated Corrector S/TEM
}

\author{
M. A. van der Stam, P. Tiemeijer, B. Freitag, R. Erni, M. Stekelenburg, D. Hubert and J. Ringnalda \\ FEI Company, Building AAE, Achtseweg Noord 5, Eindhoven, The Netherlands
}

At the last M\&M meeting, the new Titan series of dedicated aberration corrected (scanning) transmission electron microscopes were introduced. As was discussed then, and has been proven by other installations, the stability of the platform is the crucial success factor in the sub-Ångström performance of a corrected S/TEM. The correction of spherical aberration of magnetic lenses of microscopes is possible by using correctors based on multipole optics which allows for significantly improved resolution in TEM and STEM [1-4]. For the situation where the aberrations are corrected on the probe [5] (condenser lens correction), the probe can be improved in size and current density by using the corrector, however the stability of this probe to be suitable for sub-Ångström analysis is influenced mainly by the performance and stability of the microscope as well as the environment it is placed in. The right design helps the right performance.

At FEI Company, the theory of improving the spatial resolution capabilities through a new and corrector dedicated column was put into practice through Titan, a system which has a variable accelerating voltage between $80 \mathrm{kV}$ and $300 \mathrm{kV}$. By design, the following aspects were addressed: ultimate stability and ultimate flexibility. Energy resolution can be improved using a monochromator, which has been implemented on FEI Tecnai 200kV series instruments [6]. In this paper we present new data from a compact $80-300 \mathrm{kV}$ monochromator module for Titan S/TEM. One of the key design features is that the monochromator electronics are all fully contained within the FEG electronics, and therefore no second high tension tanks are required. This is done from a reliability and performance aspect, since dual tank systems are much more difficult to tune and have significant issues with 'echo' flashovers.

Traditionally, the objective lens was the most important aspect in terms of resolution and tilt capabilities [1], however this requirement no longer plays a significant role in corrected systems. The only reason to use a restrictive pole piece gap lens is if the base system has information limit restrictions, and the narrow pole piece gap lens offers slightly better information limit and therefore better resulting resolution after the corrector is fitted. The Titan systems have unsurpassed information limit even with a $6 \mathrm{~mm}$ pole piece gap lens, allowing tomography, cryo and heating experiments to be carried out with conventional $3 \mathrm{~mm}$ samples.

The addition of a third full condenser lens now means that with a monochromator, even when selecting the high resolution energy slit, the spatial resolution of the system will be around 1 Ångström in combination with a Cs corrector on the probe. While this sounds straightforward, it should be noted that significant integration and software effort is required to make the combination function well. With the extra condenser lens, the flexibility exists to vary the convergence angle while keeping the spot focused; as is setting up extremely small but narrow parallel beams. The illumination can be kept perfectly parallel while changing the exposed area of the sample, which is key for use with a condenser aberration corrector, since if this is not the case, the aberration corrector would have to be re-tuned whenever any changes are made to the condenser system. Since 
the Titan systems are built for multi-user facilities, such complex and frequent tuning requirements would be unacceptable. The hardware design allows fast and reproducible switching of modes, magnifications and techniques. Latest performance and application results from the probe corrected system, the image corrected system, the monochromator system as well as a combination of these technologies will be presented.

The authors thank the employees of CEOS $\mathrm{GmbH}$ for their contributions to making the next generation of corrector technology on Titan a success. The Titan platform is the basis of the TEAM project of the USA Department of Energy with the goal to arrive at 0.5 Ångström resolution in TEM and STEM on a single system, in combination with a $\mathrm{Cc}$ corrector developed in co-operation with CEOS GmbH.

\section{References}

[1] O. Scherzer, Z. Physik 101 (1936) 593.

[2] M. Haider et al., Nature 392 (1998) 768.

[3] M. Haider, G. Braunhausen, and E. Schwan, Optik 99 (1995) 167.

[4] P.E. Batson et al., Nature 418 (2002) 617.

[5] B. Freitag et al., Ultramicroscopy 102 (2005) 209.

[6] P.C. Tiemeijer, Inst. Phys. Conf. Ser. 161 (1999) 191.

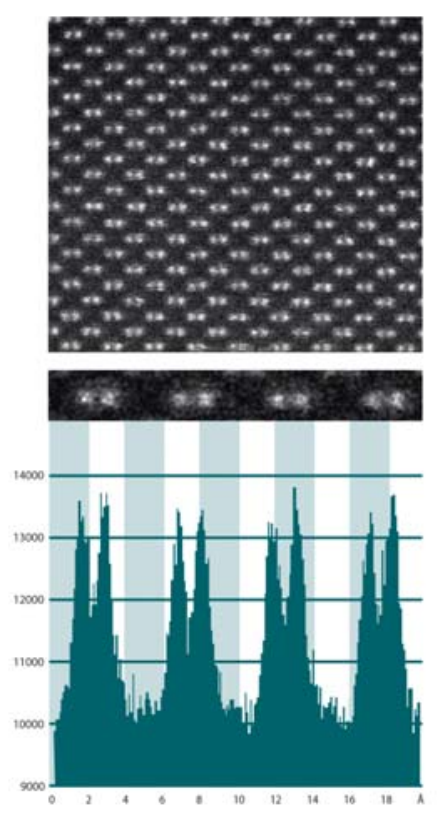

Fig. 1. Atomic resolution HR-STEM image of $\mathrm{Si}<110>$ using Titan's Cs probe corrector. The intensity profile across the dumbbell structure is shown at the bottom: Atoms are clearly resolved

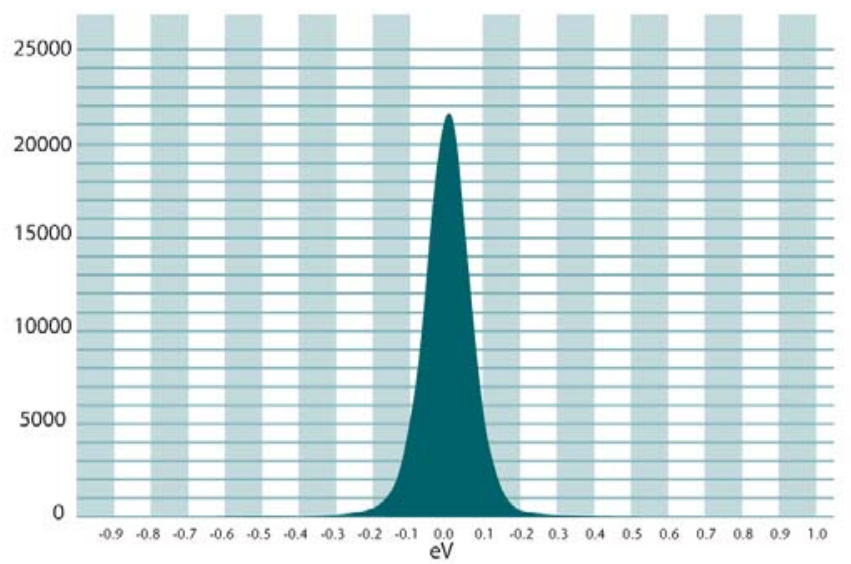

Fig. 2. Energy resolution at $300 \mathrm{kV}$ using FEI proprietary new monochromator design is better than $0.2 \mathrm{eV}$ with 1 second exposure time. 